Ann. Sci. forest., 1980, 37 (3), 173-188.

\title{
Production d'azote minéral dans divers types de landes du Massif Central
}

\author{
M. BONNEAU \\ avec la collaboration technique de M. ADRIAN, D. VAIRELLES \\ Station de Recherches sur les Sols forestiers ef la Fertilisation, I.N.R.A., \\ Centre National de Recherches forestières \\ Champenoux 54280 Seichamps
}

\begin{abstract}
Résumé
Dans des études antérieures, il a été montré que la nutrition azotée des plantations d'épicéa du Massif Central est très mauvaise à haute altitude, assez faible dans les landes à callune de moyenne alfitude et moyenne dans les landes à fougère.

On a cherché à expliquer ces faits par le potentiel de minéralisation de l'azote, soit en éfuve, soit sur le terrain. Le potentiel de minéralisation à l'étuve, variable avec la saison de prélèvement et beaucoup plus élevé que la minéralisation in situ, ne fournit pas d'explications suffisantes. II semble que la disponibilité en azote nitrique, probablement plus faible dans les landes à callune que dans les landes à fougère, et très faible en début de saison de végétation à haute altitude, puisse exercer une influence notable sur le niveau de nutrition azotée des différentes stations.
\end{abstract}

\section{Introduction}

Au cours de travaux antérieurs (Bonneau, 1972), la nutrition de jeunes plantations d'épicéas du Massif Central a été étudiée dans différentes stations. A côté de déficiences en phosphore, presque généralisées et parfois déterminantes (stations de type $c$ ci-dessous), la nutrition azotée a également retenu notre attention. De ce point de vue, les stations étudiées peuvent se rattacher à quatre types principaux :

a. - Stations d'altitude (au-dessus de $1200 \mathrm{~m}$ ), sur roche-mère

basaltique ou andésitique : l'alimentation en azote est assez déficiente.

$$
\text { b. - Stations d'altifude sur granite }
$$

La nutrition en azote est extrêmement faible : les aiguilles des jeunes épicéas contiennent généralement moins de 1 p. 100 d'azote.

c. - Stations à callune d'altitude moyenne ou faible $(500-1000 \mathrm{~m})$

Alimentation azotée d'abord correcte tant que l'effet du travail du sol se fait sentir, mais devenant médiocre entre 5 et 10 ans. 


\section{d. - Stations à fougère, d'altitude moyenne ou faible}

L'alimentation azotée, plutôt meilleure que dans les landes à callune, s'affaiblit cependant également lorsque la plantation vieillit.

II était intéressant, pour compléter ces observations, d'étudier la fourniture d'azote minéral par le sol, afin notamment de chercher à expliquer les variations d'alimentation d'une station à l'autre.

\section{I. - Matériel et méthodes}

Les recherches ont été conduites en plusieurs épisodes, de 1969 à 1975.

A. - Fin juin 1969 et début juin 1970, on a prélevé les horizons $A_{1}$ des stations suivantes:

\section{1. - Station d'altifude sur basalte}

Lac de Guéry (pelouse d'altitude). Le prélèvement a été effectué au fond de profondes raies de décapage où sont plantés les jeunes épicéas, donc dans une zone profonde de l'horizon $A_{1}$ initial qui atteint $30 \mathrm{~cm}$.

\section{2. - Stations d'altitude sur granite}

Périmètre de reboisement d'Arzenc de Randon.

Sur granite porphyroïde de Margeride et lande à myrtille, canche flexueuse ef callune. Le prélèvement a été également effectué au fond de raies de décapage et correspond donc à la couche inférieure de l'horizon $A_{1}$ entre 15 et $25 \mathrm{~cm}$ de profondeur. Deux échantillons ont été constitués, l'un à partir d'un sol témoin (Arzenc T), l'autre d'un sol chaulé (Arzenc Ca).

Périmètre « de Chalus».

On a prélevé à une centaine de mètres du reboisement d'Arzenc de Randon le même type de sol, mais dans des raies moins profondément décapées, donc dans une partie plus superficielle de l'horizon $A_{1}$.

\section{3. - Stations d'altitude moyenne à callune}

Douze stations différentes ont été étudiées, dont trois où les plantations avaient été faites sans travail du sol (Pradines, Chaumeil, Neypoux) et neuf où la callune avait été détruite par bandes grâce à un passage de rotovator (La Sagne, Péret-Bel-Air, Davignacq, Razès, St-Sétiers, Clédat 1, Clédat 2, Larfeuil). Elles se trouvent sur le plateau de Millevaches, sauf Razès qui esł située à l'extrémité Ouest des Monts d'Ambazac. Les prélèvements ont été faits dans les bandes décapées, ou bien, lorsqu'il n'y avait pas de travail mécanique du sol, à proximité d'un plant, après arrachage de la callune.

\section{4. - Stations d'altitude moyenne à fougère}

Neuf stations du Plateau de Millevaches ont été choisies : Chamberet, La Sagne, Les Géants, Pradines, Davignacq, Chaumeil, Valéoux, Grand-Grammont, Clédat. 
Plusieurs d'entre elles sont situées dans les mêmes périmètres de reboisement que les stations à callune déjà citées, parfois à quelques dizaines de mètres seulement.

II s'agit dans fous les cas de sols bruns acides très désaturés et très humifères. Le rapport $\mathrm{C} / \mathrm{N}$ est indiqué au tableau $\mathrm{I}$. Il est assez faible pour les stations d'altitude et pour les landes à fougère, plus élevé en général pour les landes à callune.

Après le prélèvement tous les sols ont été rapportés au laboratoire dans une glacière portative. Sans séchage préalable et sans tamisage, pour éviler une évolution de la teneur en azote minéral, on a dosé l'azote nitrique et l'azote ammoniacal. L'humidité de la terre fine a été déterminée sur une partie de l'échantillon, tandis que les cailloux contenus dans la terre ayant servi à l'extraction de l'azote minéral étaient récupérés et pesés : on peut ainsi calculer le poids de terre fine sèche sur lequel a porté l'analyse. Une troisième partie de l'échantillon est mise en incubation après tamisage, en double exemplaire, pendant 6 semaines à $22^{\circ} \mathrm{C}$ et à une humidité légèrement inférieure à la capacité au champ, dans le but d'obtenir la «production potentielle » d'azote minéral.

B. - En 1971, on a également pratiqué des incubations, suivant la même méthode, sur trois stations seulement : Chalus, Razès, Valéoux (trois stations de type différent).

C. - En 1975, nous avons repris des investigations plus détaillées sur trois stations représentant également des types différents : Chalus (altitude sur granite), St-Sétiers (lande à callune travaillée), Chamberet (lande à fougère).

a) Des prélèvements d'horizons $A_{1}$ et $(B)$ ont été effectués en mai et août, comme précédemment, en vue d'effectuer des incubations à l'étuve.

b) On a aussi, chaque mois, de mai à août, effectué des mesures de la minéralisation in situ dans les horizons $A_{1}$, suivant une technique imitée de celle de Lemée (1967). On réalise à partir de cinq à huit prélèvements individuels un échantillon homogène de $A_{1}$ qui sert également à l'incubation en étuve. Sur une fraction de cet échantillon on dose, suivant la technique indiquée ci-dessous, la teneur initiale en $\mathrm{NH}_{4}$ et $\mathrm{NO}_{3}$. On remplit avec l'échantillon trois cylindres en CPV qui sont ensuite réintroduits dans le sol, réhumidifiés jusqu'au voisinage de la capacité au champ et recouverts d'une soucoupe renversée pour empêcher la pluie d'y pénétrer et éviter l'évaporation. Un mois plus tard on prélève à nouveau le contenu des cylindres pour y doser l'azote ammoniacal et nitrique et déterminer, par différence avec les valeurs initiales, la production in situ d'azote minéral pendant le mois écoulé. Dans les tableaux de résultats, les valeurs de production sont des valeurs corrigées par extrapolation linéaire à 6 semaines afin qu'elles puissent être comparées avec les données de l'incubation à l'étuve. Cette extrapolation n'implique pas que la production d'azote minéral soit un phénomène linéaire (il a été en fait vérifié que si cette linéarité est assez vraie pour la production d'azote nitrique, celle d'azote ammoniacal, par suite notamment des phénomènes de réorganisation, est beaucoup plus irrégulière) ; le résultat du calcul ne représente donc qu'une estimation relativement grossière de la valeur qui aurait été obtenue en 6 semaines, mais permet cependant un parallèle avec la production en étuve, d'autant plus que, comme il sera indiqué plus loin, l'écart entre production in situ et au laboratoire est considérable.

Nos stations d'étude étant à plusieurs centaines de kilomètres du laboratoire, il a paru nécessaire d'éviter, mieux que les années antérieures, les inconvénients d'une 
évolution de la teneur en azote minéral en cours de transport, en procédant à une extraction immédiate de l'azote ammoniacal et nitrique. Deux fractions de $150 \mathrm{~g}$ de l'échantillon prélevé sont pesées et transférées aussitôt dans des flacons contenant $300 \mathrm{ml}$ de liquide d'extraction : KCl Nadditionné de merseptyl (1 p. 1000$)$ pour l'azote ammoniacal, $\mathrm{CuSO}_{4} \mathrm{~N} / 50$ pour l'azote nitrique ; chaque échantillon fait ainsi l'objet de trois extractions (trois répétitions). Après agitation de quelques heures, on décante et on filtre l'extrait qu'on conserve dans un frigidaire de camping branché sur la batterie de la voiture. On garde le sol afin de récupérer et peser les cailloux dès le retour au laboratoire. Un échantillon de terre est conservé en tube fermé afin de déterminer également à l'arrivée l'humidité au moment du prélèvement.

c) Certaines de ces minéralisations in situ ont été réalisées de manière croisée afin de mettre en lumière d'éventuels effets climatiques :

- des cylindres contenant du sol de Chamberet (moyenne altitude) ont été installés dans la station de Chalus (haute altitude) en mai eł prélevés en juin et juillet (après respectivement 1 mois et 2 mois d'incubation in situ) ;

- des cylindres contenant du sol de Chalus ont été installés en mai et juin à St-Sétiers et à Chamberet; ils ont été prélevés pour analyse 1 mois après, en juin ef juillet.

d) La croissance juvénile dans la station d'altitude de Chalus a éfé frès mauvaise, et s'accompagne d'une très mauvaise alimentation en azote qui se traduit par une forte chlorose. Mais on peut remarquer dans le boisement voisin d'Arzenc de Randon, où le comportement est en moyenne aussi mauvais, un petit nombre d'épicéas nettement plus grands et plus verts. Des échantillons de racines y ont été prélevés pour isoler d'éventuels partenaires mycorhiziens de qualité exceptionnelle, mais ceffe tentative a finalement échoué. En mai, juin, juillet et août on a prélevé des échantillons de sols au pied de 3 de ces arbres remarquables : ils ont, comme les autres prélèvements, fait l'objet de détermination de $\mathrm{N}-\mathrm{NH}_{4}$ et $\mathrm{N}-\mathrm{NO}_{3}$ au moment du prélèvement, 1 mois plus tard après séjour in situ dans des cylindres et, pour les prélèvements de mai et août, 6 semaines plus tard a près incubation à l'étuve. Ces microstations sont notées Arzenc + . C'est la moyenne de teneur en azote minéral de ces trois échantillons qui est indiquée dans les tableaux.

La détermination au laboratoire de l'azote ammoniacal et nitrique a été faite de la manière suivante :

- azote ammoniacal : en 1969, 1970 et 1971 par distillation. En 1975, par dosage en colorimétrie aufomatique Technicon : le phénate de soude en présence d'hypochlorite de sodium donne un composé diazo bleu, colorimétré à $625 \mathrm{~nm}$;

- azole nitrique : par dosage colorimétrique à $420 \mathrm{~nm}$ du composé nitré obtenu par action de l'acide phénol-disulfonique.

\section{II. - Résultats}

Le tableau I indique les teneurs maximales et minimales en azote ammoniacal ef nitrique observées in situ dans les différentes stations au cours des diverses années d'observation ; le tableau 2 indique les quantités maximales et minimales d'azote ammoniacal, nitrique et minéral tolal libérées au cours des incubations au laboratoire. En effet, le taux de minéralisation de l'azote organique se montre extrêmement fluc- 
tuant suivant les dates de prélèvement ; cela a déjà été constaté dans d'autres études (Bonneau, 1971 ; Popovic, 1971). La plus forte production relevée en étuve dans les conditions optimales d'humidité et de température peut donc représenter un potentiel maximum de minéralisation. La libération maximale d'azote nitrique ne se trouve pas forcément réalisée au cours de la même incubation que le maximum de $\mathrm{N}-\mathrm{NH}_{4}$ ou d'azote minéral total. II nous a cependant semblé que le potentiel était le mieux représenté par les maxima, même s'ils sont indépendants les uns des autres, en particulier pour mettre en valeur le potentiel maximal de nitrification. Il ne faut donc pas s'étonner si au tableau 2 la production d'azote minéral total est, dans quelques cas, différente de la somme de $\mathrm{N}-\mathrm{NH}_{4}$ et $\mathrm{N}-\mathrm{NO}_{3}$. La figure 1 permet de visualiser ce potentiel maximum, en p. 100 du stock d'azote organique, pour les différentes stations. Des maxima plus

\section{TABLEAU ।}

Teneurs maximales ef minimales en azofe minéral observées, in situ, dans les horizons $A_{1}$ de différentes landes du Mossif Central

Maximum and minimum mineral nitrogen contents, in situ, of $A_{1}$ horizons

\begin{tabular}{|c|c|c|c|c|c|c|c|c|}
\hline & \multirow{3}{*}{$C / N$} & \multirow{3}{*}{$\begin{array}{l}N \\
\text { org. } \\
\text { total } \\
\text { p. } 100\end{array}$} & \multirow{2}{*}{\multicolumn{2}{|c|}{$\frac{\mathrm{N}-\mathrm{NH}_{4}}{\mathrm{mg} / 100 \mathrm{~g}}$}} & \multirow{2}{*}{\multicolumn{2}{|c|}{$\frac{\mathrm{N}-\mathrm{NO}_{3}}{\mathrm{mg} / 100 \mathrm{~g}}$}} & \multirow{2}{*}{\multicolumn{2}{|c|}{$\frac{\mathrm{N}-\mathrm{NH}_{4}+\mathrm{N}-\mathrm{NO}_{3}}{\mathrm{mg} / 100 \mathrm{~g}}$}} \\
\hline & & & & & & & & \\
\hline & & & $\min$. & $\max$. & min. & $\max$. & $\min$. & $\max$. \\
\hline \multicolumn{9}{|l|}{ Stations d'altifude } \\
\hline $\begin{array}{l}\text { Arzenc } . . . \ldots \ldots \\
\text { Arzenc Ca. } \ldots \ldots \\
\text { Chalus } \ldots \ldots \ldots \\
\text { Arzenc }+\ldots \ldots \ldots \\
\text { Guery } . . . \ldots \ldots\end{array}$ & $\begin{array}{l}12,8 \\
13,7 \\
16,2 \\
14,7 \\
12,6\end{array}$ & $\begin{array}{l}0,37 \\
0,41 \\
0,41 \\
0,75 \\
1,37\end{array}$ & $\begin{array}{l}0,13 \\
0,52 \\
0,38 \\
0,76 \\
0,54\end{array}$ & $\begin{array}{l}1,29 \\
2,24 \\
2,75 \\
2,40 \\
0,99\end{array}$ & $\begin{array}{l}0,03 \\
0,03 \\
0 \\
0,04 \\
0\end{array}$ & $\begin{array}{l}0,03 \\
0,03 \\
0,08 \\
1,46 \\
0,06\end{array}$ & $\begin{array}{l}0,25 \\
0,55 \\
0,41 \\
0,84 \\
0,40\end{array}$ & $\begin{array}{l}1,32 \\
2,27 \\
2,83 \\
3,01 \\
1,05\end{array}$ \\
\hline \multicolumn{9}{|l|}{ Stafions à callune } \\
\hline 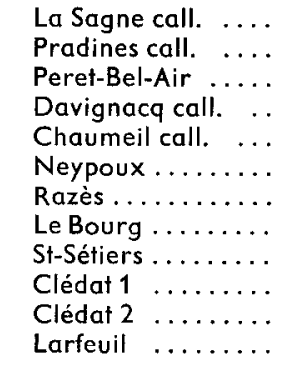 & $\begin{array}{l}18,7 \\
23,3 \\
11,0 \\
15,2 \\
22,4 \\
18,1 \\
18,7 \\
21,0 \\
22,5 \\
23,0 \\
23,0 \\
22,3\end{array}$ & $\begin{array}{l}0,38 \\
0,38 \\
0,47 \\
0,29 \\
0,66 \\
0,54 \\
0,47 \\
0,34 \\
0,41 \\
0,58 \\
0,57 \\
0,63\end{array}$ & $\begin{array}{l}0,16 \\
0,40 \\
1,19 \\
0,19 \\
0,35 \\
0,20 \\
0,25 \\
0,22 \\
0,59 \\
0,42 \\
0,54 \\
0,22\end{array}$ & $\begin{array}{l}0,52 \\
0,83 \\
1,38 \\
0,85 \\
1,36 \\
0,80 \\
2,99 \\
0,80 \\
4,65 \\
0,83 \\
1,46 \\
1,0\end{array}$ & $\begin{array}{l}0,02 \\
0,01 \\
0,19 \\
0,01 \\
0 \\
0 \\
0,01 \\
0 \\
0 \\
0 \\
0,01 \\
0\end{array}$ & $\begin{array}{l}0,06 \\
0,10 \\
0,26 \\
0,09 \\
0,07 \\
0,04 \\
0,29 \\
0,04 \\
0,97 \\
0,10 \\
0,79 \\
0,13\end{array}$ & $\begin{array}{l}0,18 \\
0,41 \\
1,38 \\
0,20 \\
0,35 \\
0,20 \\
0,26 \\
0,22 \\
0,59 \\
0,42 \\
0,55 \\
0,22\end{array}$ & $\begin{array}{l}0,58 \\
0,93 \\
1,64 \\
0,94 \\
1,43 \\
0,84 \\
3,28 \\
0,84 \\
5,62 \\
0,93 \\
2,25 \\
1,13\end{array}$ \\
\hline \multicolumn{9}{|l|}{ Stations à fougère } \\
\hline $\begin{array}{l}\text { Chamberet ....... } \\
\text { La Sagne foug. ... } \\
\text { Les Géants....... } \\
\text { Pradines foug. .... } \\
\text { Davignacq foug... } \\
\text { Chaumeil foug..... } \\
\text { Valéoux ........ } \\
\text { Grand Grammont } \\
\text { Clédat foug. ..... }\end{array}$ & $\begin{array}{l}13,2 \\
14,3 \\
11,2 \\
14,6 \\
15,4 \\
21,0 \\
13,4 \\
19,8 \\
15,4\end{array}$ & $\begin{array}{l}0,42 \\
0,36 \\
0,56 \\
0,49 \\
0,38 \\
0,65 \\
0,29 \\
0,57 \\
0,58\end{array}$ & $\begin{array}{l}0,50 \\
0,38 \\
0,59 \\
0,41 \\
0,44 \\
0,84 \\
0,10 \\
0,34 \\
0,17\end{array}$ & $\begin{array}{l}1,99 \\
1,19 \\
0,70 \\
0,82 \\
1,08 \\
1,51 \\
0,95 \\
0,48 \\
1,21\end{array}$ & $\begin{array}{l}0,02 \\
0,22 \\
0 \\
0,01 \\
0 \\
0,01 \\
0 \\
0,04 \\
0\end{array}$ & $\begin{array}{l}0,09 \\
0,22 \\
0,07 \\
0,01 \\
0,02 \\
0,08 \\
0,01 \\
0,07 \\
0,18\end{array}$ & $\begin{array}{l}0,54 \\
0,60 \\
0,59 \\
0,42 \\
0,44 \\
0,85 \\
0,10 \\
0,41 \\
0,17\end{array}$ & $\begin{array}{l}2,0 \\
1,41 \\
0,77 \\
0,83 \\
1,10 \\
1,59 \\
0,96 \\
0,52 \\
1,39\end{array}$ \\
\hline
\end{tabular}


élevés ont pu occasionnellement être observés mais nous nous sommes limités, dans le tableau 2, aux seules années 1969 ef 1970 où toutes les stations avaient été testées simultanément (sauf pour Arzenc + où les résultats se rapportent à 1975).

Les tableaux 3 et 4 regroupent les résultats obtenus pour les stations étudiées plus en détail en 1975, soit à l'air libre, soit dans les cylindres (incubation in situ), soit en incubation à l'étuve, à différentes périodes de l'été. On y a également indiqué, outre les productions d'azote minéral (tabl. 4), les quantités d'azote minéral accumulées en fin de période d'observation (tabl. 3) cette accumulation donnant, peut-être mieux que la production, une idée des possibilités d'alimentation des jeunes plants.

Le tableau 5 donne les résultats des incubations «croisées » in situ.

TABLEAU 2

Productions maximale ef minimale d'azote minéral obtenues en incubation de 6 semaines au laboratoire (Horizons $\left.A_{1}\right)$ Highest and lowest values of mineral nitrogen production obtained in a 6 week laboratory incubation for $A_{1}$ horizons

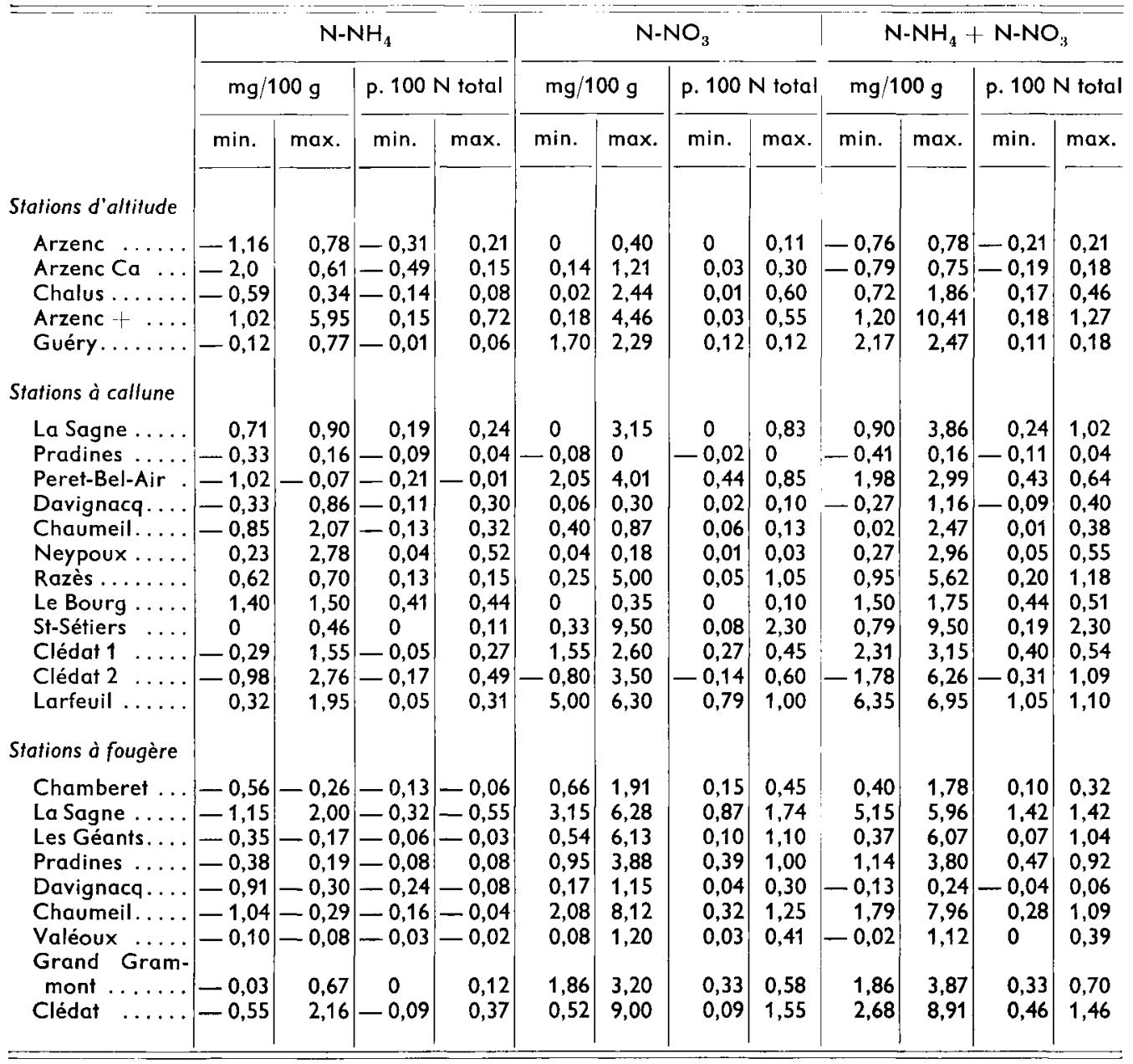




\section{1. - Teneur du sol en azote minéral in situ}

L'azote ammoniacal est peu abondant ef les valeurs maximales dans l'horizon $A_{1}$ n'excèdent que rarement $2 \mathrm{mg}$ pour $100 \mathrm{~g}$ de sol ; pour la plupart des stations, elles ont été observées fin juin 1969. Les teneurs minimales varient de 0,1 à $1 \mathrm{mg}$ avec une teneur moyenne de l'ordre de 0,5 (tabl. I) et ont généralement été relevées au début de juin 1970.

L'azote nitrique en $A_{1}$ est encore moins abondant ; la teneur minimale est fréquemment nulle ef bien souvent le maximum atteint à peine $0,1 \mathrm{mg}$ pour $100 \mathrm{~g}$, soit $1 \mathrm{~kg} / \mathrm{ha}$. On doit noter cependant quelques pointes exceptionnelles qui atteignent ou dépassent $1 \mathrm{mg} / 100 \mathrm{~g}(10 \mathrm{~kg} / \mathrm{ha})$. La valeur la plus élevée est observée dans un des échantillons d'Arzenc + , ce qui laisse supposer l'existence de microsites exceptionnellement favorables.

A un instant donné la teneur du sol en azote nitrique ne représente qu'une fraction très faible de l'azote minéral total : 3 à 8 p. 100.

Le tableau 3 n'indique pas, dans les horizons $A_{1}$, pendant la période estivale, de variation systématique de la richesse du sol en azote minéral en fonction du temps.

Les horizons (B) sont moins riches en azote ammoniacal que les horizons $A_{1}$ (tabl. 3) mais les teneurs en nitrates sont du même ordre. Si l'on estime qu'un jeune plant prospecte une quarantaine de $\mathrm{cm}$ de profondeur $\left(20 \mathrm{~cm}\right.$ en $A_{1}$ ef $20 \mathrm{~cm}$ en (B)), ef compte tenu des densités apparentes plus élevées en (B) qu'en $A_{1}$, on peut tabler sur des réserves variant, au cours de la saison de végétation, de 10 à $40 \mathrm{~kg}$ d'azote ammoniacal et 1 à $5 \mathrm{~kg}$ d'azote nitrique.

Les teneurs en azote minéral ne permettent pas de différencier entre elles les différentes stations. On peut noter cependant la teneur relativement élevée en azote nitrique et minéral total de Arzenc + + , St-Sétiers, Clédał 2, La Sagne (fougère).

\section{2. - Minéralisation potentielle (incubation à l'étuve)}

Les résultats sont extrêmement variables, pour une même station, suivant l'année ou la date des prélèvements.

Les tableaux 3 et 4 (colonnes de droite) montrent, pour 1975, une plus forte accumulation en valeur absolue et une plus forte production pour les incubations d'août-septembre. Ceci est particulièrement net pour la production d'azote nitrique à moyenne altitude (St-Sétiers, Chamberet) et à la fois pour l'ammonium et les nitrates dans les stations de haute altitude. A Chalus, la production potentielle d'azote nitrique apparaît comme particulièrement faible en début de saison; elle est un peu meilleure à Arzenc + , en valeur absolue $(\mathrm{mg} / 100 \mathrm{~g})$, grâce à une teneur plus élevée du sol en azote organique, mais le taux de production de $\mathrm{N}-\mathrm{NO}_{3}$ par rapport à $\mathrm{N}$ total est aussi bas qu'à Chalus (łabl. 4).

La figure 1 montre que la discrimination est assez mauvaise entre les quatre grandes catégories de stations, en ce qui concerne le potentiel maximum de production d'azote minéral. Les stations d'altitude se classent cependant dans la zone de faible accumulation potentielle de nitrates, les deux plus mauvaises, Guéry et Arzenc de Randon correspondant à des fonds de raies profondément décapées. Chalus, où l'horizon $A_{1}$ est resté presque intact, se classe presque aussi bien que la moyenne des stations de moindre altitude ; Arzenc + a également une situation exceptionnellement favorable 


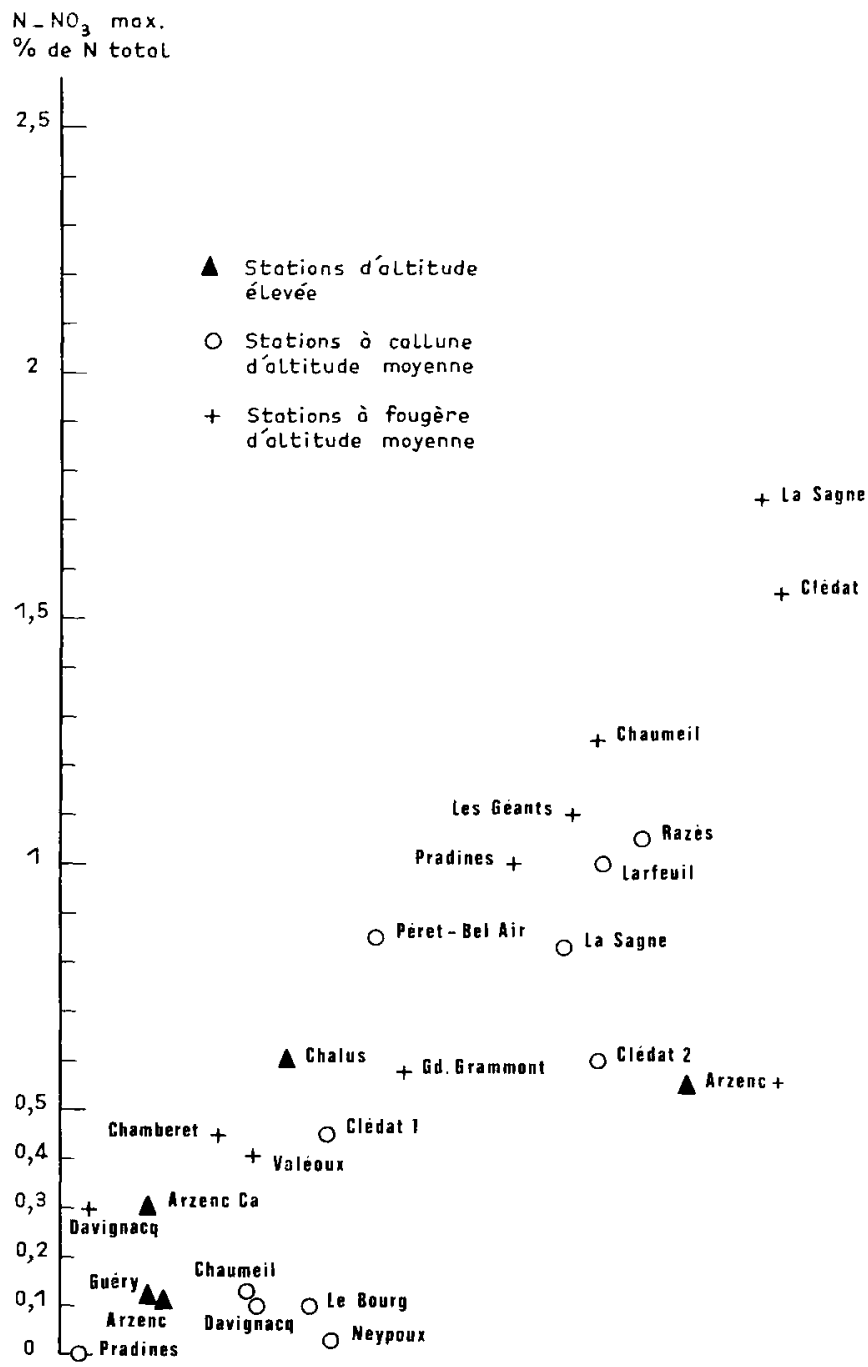

St. Setiers 0

$$
\begin{aligned}
& \text { Stotions d̀ collune } \\
& \text { d'altitude moyenne } \\
& \text { Stations ò fougère } \\
& \text { d'oltitude moyenne }
\end{aligned}
$$$$
+ \text { La Sagne }
$$$$
\text { + Clèdat }
$$$$
\text { + Chaumeil }
$$$$
\stackrel{\text { Les fieants }+}{\text { O Razes }}
$$$$
\text { Peret - Bel Air }
$$$$
\text { La Sagne }
$$

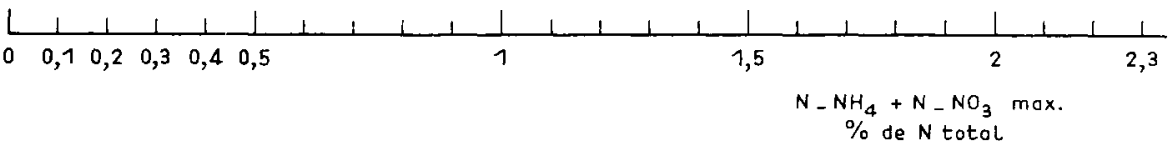

FIG. 1. - Production maximale de $\mathrm{N}-\mathrm{NO}_{3}$ ef $\mathrm{N}$ minéral, en p. 100 de l'azote total obtenue en incubation de 6 semaines à l'étuve.

Highest production of $\mathrm{NO}_{3}-\mathrm{N}$ and mineral $\mathrm{N}$, in p. 100 of total nitrogen, oblained in a 6 week laboratory incubation.

parmi les stations d'altitude élevée, avec une nitrification assez importante et un taux de minéralisation global qui est parmi les plus élevés de l'ensemble des stations étudiées. On peut noter encore l'amélioration relative assez nette due au chaulage à Arzenc de Randon, sur le plan de la nitrification. 
Les stations à callune ef les stations à fougère se mêlent sur le graphique, avec cependant une majorité de landes à fougère au-dessus de $1 \mathrm{p}$. $100 \mathrm{de} \mathrm{N}-\mathrm{NO}_{3}$. Cinq landes à callune, parmi lesquelles les trois où il n'y a pas eu de travail du sol, ne manifestent qu'un potentiel très minime de nitrification, du même ordre que celui des stations d'altitude. Ainsi apparaît l'effet très important, déjà signalé (Bonneau, 1971), du travail du sol et de l'éradication de la callune sur le potentiel de minéralisation de l'azote organique.

La faible différence entre landes à callune travaillées ef landes à fougère correspond à l'observation de terrain suivante, assez fréquente : après travail du sol, ef même parfois sans travail du sol, la fougère se réinstalle avec vigueur dans certaines landes à callune. Il y aurait de " fausses landes à callune » qui correspondent simplement au résultat d'un pâturage intensif passé qui a fait régresser artificiellement la fougère. Inversement, parmi les landes à fougère, Davignacq, Chamberet ef Valeoux ne manifestent que des potentiels de minéralisation et de nitrification très médiocres.

\section{3. - Production d'azote minéral in situ (tabl. 3 et 4 )}

On peut avoir une idée assez valable de la production nette d'azote ammoniacal de l'horizon $A_{1}$ (minéralisation diminuée de la réorganisation et de la nitrification) par la différence des teneurs de cet horizon entre deux prélèvements successifs : les emplacements où sont prélevés les échantillons ont été débarrassés de leur végétation à l'automne 1974 ; il n'y a donc eu aucun prélèvement ef l'azote ammoniacal migre peu. Dans ces conditions, les teneurs finales en ammonium à l'air libre el dans les cylindres devraient être voisines. Or, on note des écarts très sensibles : la quantité d'ammonium dans les cylindres est généralement moindre en juin et supérieure en août. Au début de l'été, au moins, la différence devrait être en faveur des cylindres, dans lesquels l'humidité a été réajustée à la capacité de rétention. L'écart constaté provient vraisemblablement de la mauvaise conductivité thermique des cylindres à l'intérieur desquels le sol se réchauffe moins vite, en début d'été, qu'à l'air libre. Le fonctionnement des incubations in situ ne s'est donc pas révélé satisfaisant (tabl. 3 et 4).

On peut cependant remarquer que, même en plein été, la minéralisaticn in situ, dans les cylindres, reste très inférieure à la minéralisation à l'étuve. Le potentiel d'activité des humus ne trouve donc pas, dans les conditions naturelles, sa pleine expression.

La nitrification dans les cylindres (colonnes $\mathrm{B}$ ) est de plus en plus active à mesure que la saison s'avance ef on retrouve donc là une évolution parallèle à celle de la minéralisation à l'étuve.

A cause des réserves qui viennent d'être faites vis-à-vis des incubations in situ, ef aussi parce que les conditions climatiques réelles des stations de moyenne altitude et de haute altitude peuvent être très différentes, il est difficile de comparer entre elles les activités des humus in situ, sauf si les stations sont très proches. Cette condition étant remplie pour Chamberet et St-Sétiers, on peut ainsi constater que, alors que la minéralisation potentielle en étuve, en début de saison, est sensiblement égale pour l'horizon $A_{1}$ de la lande à fougère (Chamberet) et pour celui de la lande à callune (St-Sétiers), la nitrification en cylindres in situ est beaucoup plus faible dans la première station et ne remonte que progressivement. On peut penser que la situation topographique de la station de Chamberet, en pied de pente et en versant nord, entraîne un réchauffement plus tardif. L'ombrage de la fougère, qui s'est réinstallée avec vigueur à partir de fin 
TABLEAU 3

Teneurs en azote minéral en $\mathrm{mg} / 100 \mathrm{~g}$ de sol à différentes dates et après différents traitements
A) in situ
B) après incubation en cylindres sur le terrain
C) après incubation de 6 semaines au laboratoire

Content in mineral nitrogen, in $\mathrm{mg}$ for $100 \mathrm{~g}$ of soil, at different dates and after different treatments
A) in the open field
B) after incubation in boxes in the field
C) after a 6 weeks laboratory incubation

\section{Arzenc + $\mathrm{A}_{1}$}

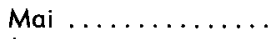

Juin ...........

Juillet. . . . . . . . .

Août............

0,75

0,89

Septembre ......

0,75

$N$ total

p. 100

du sol sec

Chalus $A_{1}$

Mai $\ldots \ldots \ldots \ldots$.

Juin ...........

Juillet. . . . . . . . .

Août. . . . . . . . . .

Septembre ........

Chalus B

Mai ............

Juin...$\ldots \ldots \ldots$

Juillet. . . . . . . . .

Août............

St-Sétiers $A_{1}$

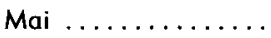

Juin . . . . . . . . .

Juillet. . . . . . . . . .

Août.............

Septembre ........

1,90
1,74
1,33
1,43

0,13

0,11

0,05

0,56

1,19

2,43

0,68

0,26

1,71

0,77

7,53

4,99

1,82

2,40

0,07

2,75

0,05

0,08

1,04

1,08
1,49
1,94

0,07

0,16

0,73

C

$\begin{array}{lll} & 0,89 & 0,09 \\ & 0,54 & 0,03 \\ 0,26 & 1,00 & 0,05 \\ 0,25 & 1,53 & 0,02\end{array}$

0,56

0,49

0,43

0,41

2,54

0,10

$2,04 \quad 0,06$

$2,20 \quad 0,10$

$1,71 \quad 0,04$

1,35

0,22

4,66

1,09

2,19

0,13

0,37

3,90

\section{St-Sétiers B}

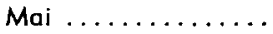

Juin . . . . . . . .

Juillet. . . . . . . . .

Août. .

0,09

0,16

$0,73 \quad 0,04$

0,16

0,43

0,05

0,18

0,70

0,03

Chamberef $A_{1}$

Mai ...........

0,54

Juin $\ldots \ldots \ldots \ldots \ldots$

0,56

Juillet. . . . . . . . . .

0,55

1,36

1,11

0,09

1,96

0,02

0,48

0,87

0,04

Septembre

0,03

0,69

1,47

0,08

0,07

2,71

1,32

1,31

2,20

2,13

\section{Chamberet B}

Mai ............

Juin ............

Juillet. . . . . . . . . .

0,19

1,02

0,08

0,12

0,51

0,03

Août.

0,12

0,42

0,04

0,19

0,63 


\section{TABLEAU 4}

Production d'azołe minéral dans différentes conditions

A) in situ en 6 semaines

B) après incubation dans les cylindres sur le terrain

C) après incubation de 6 semaines au laboratoire

Production of mineral nitrogen in different conditions

A) in the open field

B) after incubation in boxes in the field

C) after a 6 week laboratory incubation

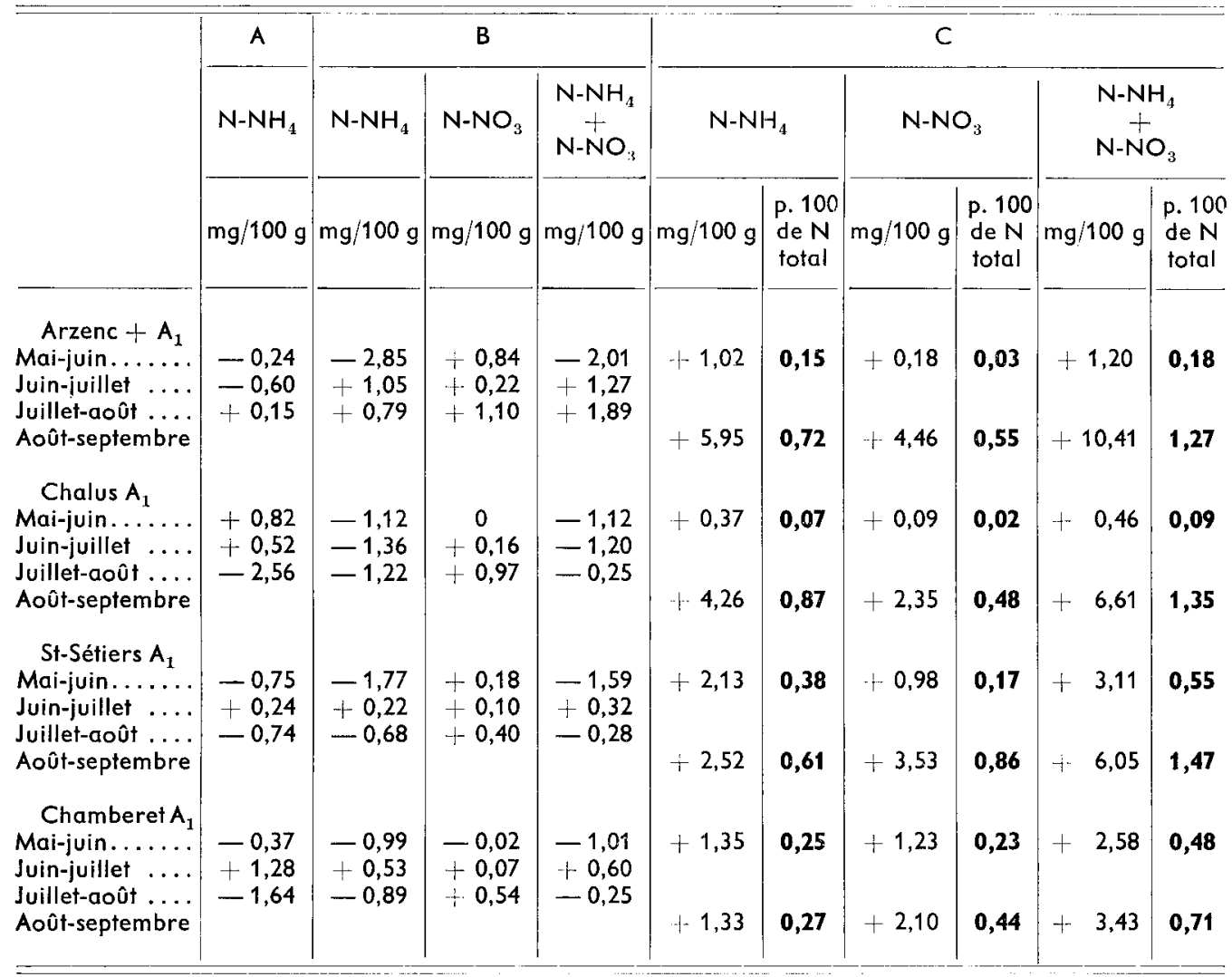

juin, peut être aussi en cause. On remarque également que les échantillons d'Arzenc + nitrifient beaucoup plus activement en début de saison que ceux de Chalus et que l'écart de production des nitrates est beaucoup plus sensible in situ qu'en incubation à l'étuve.

Les incubations in situ croisées permettent également des observations intéressantes (tabl. 5). A St-Sétiers, l'humus provenant de Chalus montre une nitrification nulle alors que celui de St-Sétiers produit une quantité appréciable d'azote nitrique. Dans la station de Chalus, l'horizon $A_{1}$ de cette station produit aussi beaucoup moins d'azote minéral, spécialement nitrique, que l'échantillon en provenance de Chamberet. L'humus de Chalus apparaît donc comme très peu actif par rapport aux autres.

Il est remarquable aussi qu'en juin-juillet, l'humus de Chalus, qui a une nitrifi- 
cation positive, bien que faible, dans sa station d'origine, ne libère pas de nitrates lorsqu'il est en incubation à Chamberet : ceci confirme l'observation, déjà faiłe plus haut, de l'effet d'exposition ou d'ombrage de cette station.

\section{TABLEAU 5}

Incubations croisées : production d'azote ammoniacal et nitrique par des horizons $\boldsymbol{A}_{1}$ provenant de diverses stations (colonne 2) et incubés in situ soit dans leur station d'origine, soit dans d'autres stations (colonne 1)

$\mathrm{N}-\mathrm{NH}_{4}$ and $\mathrm{N}-\mathrm{NO}_{3}$ production in four localities (column 1) by $\mathrm{A}_{1}$ horizons from different sites (column 2) (crossed field-incubations)

\begin{tabular}{|c|c|c|c|c|c|c|c|c|c|}
\hline \multirow{3}{*}{$\begin{array}{l}\text { Station } \\
\text { (Site) }\end{array}$} & \multirow{3}{*}{$\begin{array}{c}\text { Station-origine } \\
\text { de l'échantillon } \\
\text { (Origine-site of } \\
\text { the humus } \\
\text { sample) }\end{array}$} & \multicolumn{4}{|c|}{ Mai-juin } & \multicolumn{4}{|c|}{ Juin-juillet } \\
\hline & & \multirow{2}{*}{$\begin{array}{c}\mathrm{N}-\mathrm{NH}_{4} \\
\mathrm{mg} / 100 \mathrm{~g}\end{array}$} & \multirow{2}{*}{$\begin{array}{c}\mathrm{N}-\mathrm{NO}_{3} \\
\mathrm{mg} / 100 \mathrm{~g}\end{array}$} & \multicolumn{2}{|c|}{$\mathrm{N}-\mathrm{NH}_{4}+\mathrm{N}-\mathrm{NO}_{3}$} & \multirow{2}{*}{$\begin{array}{c}\mathrm{N}-\mathrm{NH}_{4} \\
\mathrm{mg} / 100 \mathrm{~g}\end{array}$} & \multirow{2}{*}{$\begin{array}{c}\mathrm{N}-\mathrm{NO}_{3} \\
\mathrm{mg} / 100 \mathrm{~g}\end{array}$} & \multicolumn{2}{|c|}{$\mathrm{N}-\mathrm{NH}_{4}+\mathrm{N}-\mathrm{NO}_{3}$} \\
\hline & & & & $\mathrm{mg} / 100 \mathrm{~g}$ & $\begin{array}{l}\text { p. } 100 \\
N \text { total }\end{array}$ & & & $\mathrm{mg} / 100 \mathrm{~g}$ & $\begin{array}{l}\text { P. } 100 \\
N \text { total }\end{array}$ \\
\hline $\begin{array}{c}\text { St-Sétiers } \\
\text { Chamberet } \\
\text { Chalus } \\
\text { Arzenc }+\end{array}$ & $\begin{array}{c}\text { St-Sétiers } A_{1} \\
\text { Chalus } A_{1} \\
\text { Chamberet } \\
\text { Chalus } A_{1} \\
\text { Chalus } \\
\text { Chamberef } \\
\text { Arzenc }+\end{array}$ & $\begin{array}{l}-1,77 \\
-1,30 \\
-0,99 \\
-1,53 \\
-1,12 \\
-0,28 \\
-2,85\end{array}$ & $\begin{array}{l}+0,18 \\
-0,01 \\
-0,02 \\
-0,05 \\
0 \\
+0,24 \\
+0,84\end{array}$ & $\begin{array}{l}-1,6 \\
-1,31 \\
-1,01 \\
-1,58 \\
-1,12 \\
-0,04 \\
-2,01\end{array}$ & $\begin{array}{l}-0,28 \\
=0,24 \\
=0,19 \\
=0,29 \\
=0,20 \\
=0,01 \\
-0,29\end{array}$ & $\begin{array}{r}+0,22 \\
-1,53 \\
+0,54 \\
+0,72 \\
-1,36 \\
+0,96 \\
+1,05\end{array}$ & $\begin{array}{l}+0,10 \\
+0,03 \\
+0,07 \\
0 \\
+0,16 \\
+0,90 \\
+0,22\end{array}$ & $\begin{array}{l}+0,32 \\
-1,56 \\
+0,61 \\
-0,75 \\
-1,20 \\
+1,86 \\
+1,27\end{array}$ & $\begin{array}{l}+0,06 \\
-0,28 \\
+0,11 \\
-0,16 \\
-0,21 \\
+0,33 \\
+0,17\end{array}$ \\
\hline
\end{tabular}

\section{Discussion. Conclusion}

\section{1. - Sur le plan méthodologique}

L'incubation à l'étuve ne peut guère fournir de résultats intéressants en ce qui concerne l'alimentation minérale des reboisements. En effet, ses résultats sont très fluctuants en fonction de la date et de l'année de prélèvement ; de plus, la minéralisation in situ lui reste très inférieure. Cependant, si l'on désire, en vue d'une étude théorique des humus, avoir une idée du maximum de minéralisation potentielle, le prélèvement devra être fait tardivement, début août.

La minéralisation in situ est probablement beaucoup plus intéressante en pratique, mais elle ne peut fournir des résultats exacts que si les échanges thermiques des échantillons avec le sol ambiant sont bien assurés. De ce point de vue, des cylindres épais en matière plastique ne conviennent pas ; il faut employer des cylindres métalliques à parois minces (technique de Lemée) ou des sacs en polyéthylène.

\section{2. - Sur le plan de l'alimentation en azote des reboisements}

Le tableau 6 indique la teneur foliaire en azote des jeunes épicéas des stations étudiées (sauf Clédał 2 plantée en Douglas et Arzenc + où l'analyse n'a pas été faite).

Il est manifeste qu'il n'y a pas de relation entre cette teneur en azote et le potentiel de minéralisation obtenu en étuve. Certains résultats sont même tout à fait contradic- 


\section{TABLEAU 6}

Teneur en $N$ ef $P$ foliaires totaux, en p. 100 de la matière sèche, dans les aiguilles de 1 an des jeunes épicéas des stations éfudiées (Clédat 2 ne figure pas car il s'agit d'une plantation de Douglas)

Total nitrogen and phosphorus per cent of dry weight in one-year needles of young spruce from the investigated sites (Cledat 2 is not noted because it is a Douglas plantation)

\begin{tabular}{|c|c|c|c|c|}
\hline & \multicolumn{2}{|c|}{$\mathrm{N}$} & \multicolumn{2}{|c|}{$\mathbf{P}$} \\
\hline & 1969 & 1970 & 1969 & 1970 \\
\hline \multicolumn{5}{|l|}{ Stations d'altitude } \\
\hline $\begin{array}{l}\text { Arzenc } \mathrm{T} \ldots \ldots \ldots \ldots \\
\text { Arzenc } \mathrm{Ca} \ldots \ldots \ldots \ldots \\
\text { Chalus } \ldots \ldots \ldots \ldots \\
\text { Guéry } \quad \ldots \ldots \ldots \ldots \ldots\end{array}$ & $\frac{1,09}{-}$ & $\begin{array}{l}1,06 \\
1,06 \\
1,32 \\
1,30\end{array}$ & $\frac{0,23}{-}$ & $\begin{array}{l}0,19 \\
0,23 \\
0,20 \\
0,22\end{array}$ \\
\hline \multicolumn{5}{|l|}{ Stations d̀ callune } \\
\hline 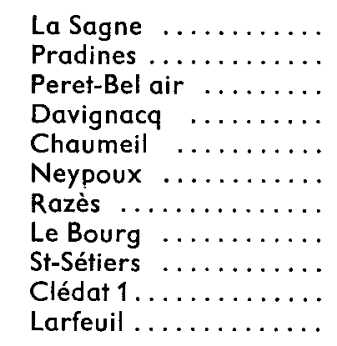 & $\begin{array}{l}1,17 \\
1,64 \\
1,75 \\
1,40 \\
1,81 \\
1,51 \\
1,83 \\
1,11 \\
2,00 \\
2,16 \\
1,10\end{array}$ & $\begin{array}{l}1,15 \\
1,56 \\
1,34 \\
1,60 \\
1,98 \\
1,27 \\
1,60 \\
1,20 \\
1,91 \\
1,47 \\
1,04\end{array}$ & $\begin{array}{l}0,09 \\
0,13 \\
0,08 \\
0,14 \\
0,18 \\
0,13 \\
0,15 \\
0,11 \\
0,14 \\
0,12 \\
0,08\end{array}$ & $\begin{array}{l}0,10 \\
0,14 \\
0,09 \\
0,16 \\
0,21 \\
0,12 \\
0,14 \\
0,15 \\
0,14 \\
0,18 \\
0,09\end{array}$ \\
\hline \multicolumn{5}{|l|}{ Stations à fougère $\ldots \ldots \ldots$} \\
\hline 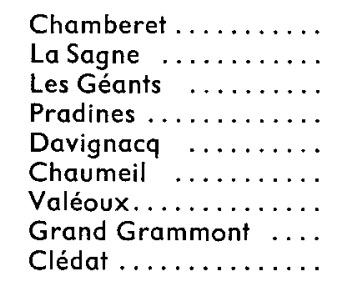 & $\begin{array}{l}1,55 \\
2,05 \\
1,67 \\
1,88 \\
2,10 \\
1,68 \\
1,99 \\
1,88 \\
1,94\end{array}$ & $\begin{array}{l}1,71 \\
1,87 \\
1,93 \\
1,37 \\
1,90 \\
1,50 \\
1,79 \\
1,76 \\
1,84\end{array}$ & $\begin{array}{l}0,19 \\
0,18 \\
0,24 \\
0,27 \\
0,26 \\
0,19 \\
0,21 \\
0,15 \\
0,23\end{array}$ & $\begin{array}{l}0,20 \\
0,20 \\
0,31 \\
0,19 \\
0,20 \\
0,15 \\
0,19 \\
0,18 \\
0,23\end{array}$ \\
\hline
\end{tabular}

toires : par exemple, les jeunes épicéas de Pradines (callune), Davignac (callune ef fougère) ont une nutrition assez bonne alors que le potentiel de minéralisation est très faible ; à l'inverse, ceux de Larfeuil et de La Sagne (callune) ont des aiguilles très paurres en azote bien que la minéralisation potentielle soit bonne dans ces stations.

Les différences dans les méthodes de travail du sol, la diversité probable du matériel génétique, peuvent contribuer à expliquer ces différences. On sait aussi que la callune exerce une influence inhibitrice sur la mycorhization ef donc sur la nutrition (Handley, 1962). La faible teneur en azote des plants de certaines stations s'explique peut-être de cette manière. On peut voir d'ailleurs, au tableau 6, qu'une mauvaise nutrition azotée va parfois de pair avec une mauvaise alimentation en phosphore (Larfeuil, La Sagne callune, Peret-Bel-air pour 1970, Le Bourg). Malheureusement, aucune étude sur la mycorhization n'a été effectuée. 
La très grande variabilité des résultats obtenus en incubation, en étuve, suivant la date de prélèvement, laisse d'ailleurs prévoir qu'aucun pronostic valable de la nutrition azotée ne peut être fondé sur ce test. Les résultats de 1975 indiquent en outre clairement que la minéralisation réelle, in sifu, est toujours très inférieure à la minéralisation potentielle. Si on voulait «expliquer » avec les meilleures chances de succès les différences d'alimentation azotée, entre les différentes stations, c'est donc à une évaluation de la minéralisation in situ qu'il faudrait avoir recours ; mais ce procédé est malheureusement beaucoup plus lourd.

Les résultats de cette étude mettent en évidence la très faible vitesse de minéralisation du stock d'azote organique dans les sols des landes du Massif Central. La minéralisation potentielle (in vitro pendant 6 semaines) n'est que de l'ordre de 1 p. 100 de l'azote organique alors qu'elle peut atteindre 2 à 5 p. 100 dans des sols forestiers de plaine (2,5 p. 100 dans une chênaie acidiphile, 4,6 p. 100 dans une hêtraie à mull-moder, d'après Lemée, 1967). Bien que dans la plupart des stations le taux de nitrification ne soit pas très faible, ce taux de minéralisation totale réduit entraîne une faible disponibilité en azote nitrique. Or, divers auteurs ont montré qu'en milieu acide l'épicéa avait une meilleure croissance en alimentation mi-nitrique, mi-ammoniacale (Evers, 1964) et absorbait préférentiellement l'azote nitrique (Hoffmann, 1966 ; Hoffmann et Fiedler, 1966). Les nitrates sont facilement entraînés dans les eaux de drainage et le sol reste pauvre si la minéralisation ne remplace pas rapidement ceux que l'eau a éliminés. Or, dans nos stations faiblement minéralisatrices ce remplacement est très lent (tabl. l) ef c'est un handicap sérieux pour la nutrition des jeunes plants dont le système racinaire est peu développé, ce qui l'empêche de suppléer facilement au manque d'azote nitrique par les ions $\mathrm{NH}_{4}^{+}$peu mobiles.

Globalement, il n'est donc pas surprenant que les stations à fougère, en majorité plus minéralisatrices, à capacité en eau plus forte et bénéficiant souvent d'apports latéraux (pieds de pente), aient une meilleure nutrition azotée puisque la profondeur du sol prospectable ralentit la perte des nitrates et que les apports latéraux peuvent en fournir.

Le problème de l'alimentation en azote nitrique se trouve ainsi soulevé. On notera aussi, à ce sujet, que le chaulage assure, à Arzenc-de-Randon, à la fois une meilleure croissance (Bonneau, 1972) ef une meilleure nitrification (fig. 1). Les microstations d'Arzenc + , nettement plus favorables, sont caractérisées par une nitrification plus précoce (tabl. 3 et 4). A conditions climatiques égales, les humus de moyenne altitude ont également une meilleure nitrification que les humus de haute altitude (tabl. 5). La disponibilité en nitrates joue donc probablement un rôle non négligeable.

On peut finalement conclure que les tests de minéralisation de l'azote, notamment ceux pratiqués au laboratoire, restent largement incapables d'éclairer complètement les différences constatées dans la nutrition azotée de l'épicéa entre les différentes stations du Massif Central, d'autant plus que la minéralisation reste très faible et très variable dans le temps. Ce manque de pouvoir discriminateur est particulièrement net à moyenne altitude entre landes à callune et landes à fougère. Cependant, la nitrification tardive et faible des stations de haute altitude peut fournir une explication partielle de la très forte carence azotée que manifestent toujours les jeunes épicéas dans ces sols.

De toute façon, d'autres facteurs doivent interférer puissamment avec la libération d'azote minéral pour déterminer le niveau de l'alimentation azotée : phosphore, alimentation en eau, mycorhization. Une preuve de l'influence limitée de l'offre d'azote est aussi donnée par la bonne réussite, à haute altitude, d'autres espèces, le pin à crochets 
notamment, dont le très bon comportement illustre la nécessité de distinguer le concept « d'absorbabilité » de l'azote de celui de « disponibilité » (Van Praag et Weissen, 1973). Laatsch et Zech (1967) ont formulé aussi l'hypothèse d'une labilité de la chlorophylle de l'épicéa en conditions climatiques et nutritives difficiles, qui aurait un effet « boule de neige " en entraînant, par l'intermédiaire d'une faible photosynthèse, une déficience de la croissance des racines, de l'absorption de l'azote et de l'effet rhizosphère souvent indispensable à une bonne nutrition.

Reçu pour publication en ocfobre 1979.

\section{Summary}

\section{Nitrogen mineralization in several kinds of heaths of the Massif Central}

It has been found in previous studies that nitrogen nutrition of young Norway Spruce plantations in the Massif Central was very poor at the highest elevations (1 200-1 $400 \mathrm{~m}$ ), rather good in bracken heaths at medium elevations and rather low in Calluna heaths at the same elevations.

In this work we attempted to find out to what extent the potential (6 weeks incubation at $22{ }^{\circ} \mathrm{C}$ ) or actual (in sifu incubation in plastic boxes) nitrogen mineralization could account for the differences in $\mathrm{N}$-nutrition between sites.

The results are not clear but the following trends can be observed : tivity.

a) The plastic boxes are not convenient for in situ incubation because of their low thermic conduc-

b) The potential rate of nitrogen mineralization varies greatly with the sampling season; it seems that sampling at the end of July gives the best chances to obtain the maximum potential rate.

c) Actual in situ mineralization is always much lower than the potential one by incubation at $22^{\circ}$.

d) The production of $\mathrm{NO}_{3}-\mathrm{N}$ is generally low in all sites.

e) Humus from high elevation sites have a much lower spring nitrification activity than humus on medium elevation sites.

f) Most of the Calluna sites of medium elevation have a lower nitrification potential and probably a lower nitrate availability than bracken sites, but there are several exceptions; nitrification is more active when Calluna has been destroyed before plantation ; after cultivation, several Calluna sites gradually changed into bracken sites and in these cases the initial vegetation at the time of afforestation was probably influenced by previous human activity (pasture, old cultivation).

g) Digging deep furrows before planting, as is frequently done in high elevation sites, causes young spruces to be planted into the lowest part of the $A_{1}$ horizon, where nitrogen mineralization is very slow.

However, it seems that the difficulties of nitrogen mineralization and nitrification cannot completely explain the very bad nitrogen nutrition in high elevation sites ( $N$-content in foliage is only about 1 p. 100).

\section{Zusammenfassung}

\section{Stickstoffmineralisation auf verschiedenen Heidestandorttypen im französischen Zentralmassiv}

Frühere Untersuchungen haben gezeigł, dass die Stickstoffernährung junger Fichtenkulturen im Zentralmassiv in den Hochlagen (1 200-1 $400 \mathrm{~m}$ ) sehr schlecht ist. In mittleren Höhenlagen ist die Stickstoffernährung auf Adlerfarnstandorten verhältnismässig gut, während sie auf Callunaheiden völlig unbefriedigend ist.

In der vorliegenden Arbeit wird versucht die beobachteten Unterschiede in der Stickstoffernährung durch die potentielle Stickstoffmineralisation (6 Wochen im Laborbrutschrank bei 22 oC) oder die tatsächliche Stickstoffmineralisierung in situ (Feldbebrütung in Plastikzylindern) zu erklären.

Obwohl die Ergebnisse keine eindeutigen Schlussfolgerungen zulassen, können die folgenden Tendenzen herausgestellt werden :

a) Plastikzylinder sind wegen ihrer schlechten Wärmeleitfähigkeit für die Feldbebrütung in situ nicht geeignet. 
b) Die potenfielle Mineralisation hängt sehr stark von der Jahreszeit der Probennahme ab. Die höchsten Werte wurden im Juli erreicht.

c) Die Feldbebrütung ergibt immer wesentliche niedrigere Werte als die Laborbebrüłung.

d) Die Nitratproduktion ist auf allen Standorten sehr gering.

e) Die Nitratproduktion im Frühjahr ist in den Hochlagen wesentlich niedriger.

f) In mittleren Höhenlagen ist die Nitrifikatiion in den Callunaheiden meist geringer als auf Adlerfarnstandorten. Eine Bodenbearbeitung erhöht die Nitrifikation. Eine Bodenbearbeitung von Callunaheiden führt oft zu einer starken Ausbreiłung des Adlerfarns und man kann daher annehmen, dass viele Callunaheiden auf ehemalig landwirtschaftlich genutzten Böden (Weide, Acker) entstanden sind.

g) Bei einem tiefen streifenweisen Abzug der Bodendecke, werden die Pflanzen im unteren Teil des $A_{1}$-Horizontes eingebracht, in dem die Mineralisierung sehr gering ist.

Die schlechte Stickstoffmineralisation und Nitrifikation erlauben jedoch nur eine teilweise Erklärung der geringen Stickstoffernährung (ca. 1 p. 100 Gesamtstickstoff in den Nadeln) auf Heidestandorten in Hochlagen.

\section{Références bibliographiques}

BONNEAU M., 1967. Minéralisation de l'azote de deux sols des Hautes-Vosges. Allure du phénomène et tentatives d'amélioration. Science du Sol (1), 19-48.

BONNEAU M., 1971. Nouvelles observations sur la minéralisation de l'azote dans deux sols des Hautes-Vosges. Science du Sol (1), 31-46.

BONNEAU M., 1972. Contribution à l'étude de la nutrition et de la fertilisation des plantations d'épicéas dans le Massif Central. Ann. Sci. Forest., 29 (1), 3-34.

ENO F., 1960. Nitrate production in the field by incubating the soil in polyethylene bags. Soil Sci. Soc. Am. Proc., 24, 277-279.

ELLENBERG H., 1977. Stickstoff als Standortsfaktor, insbesondere für mitteleuropaische Pflanzengeselleschäften. Oecol. Plant., 12 (1), 1-22.

EVERS F. H., 1964. Die Bedeutung der Stickstoffform für Wachstum und Ernährung der Pflanzen, insbesondere der Waldbaüme. Mitfeilungen des Vereins für forstliche Standortkunde und Forstpflanzenzüchtung (14), 19-37.

GERLACH A., 1973. Methodische Untersuchungen zur Bestimmung der Stickstoffnettomineralisation. Scripta geobotanica (Göttingen), 5.

HANDLEY W. R. C., 1962. Ectotrophic mycorhizal fung i and the growth of trees on calluna heathlands. Proc. 13th Congr. I.U.F.R.O. Vienne 1961, Pt 2 (1), Sect. 24/25.

HOFFMANN F., 1966. Unfersuchungen zür Stickstoffernährung junger Koniferen. II. Die Aufnahme von Ammonium und Nitratstickstoff durch Fichtensamlinge unter verschiedenen Bedingungen. Arch. f. Forstwesen, 15 (10), 1093-1103.

HOFFMANN F., FIEDLER H., 1966. Die Stickstoffernährung junger Koniferen. Biologische Rundschau, $4(3), 138-155$.

LAATSCH W., ZECH W., 1967. Die Bedeutung der Beschattung für unzureichend ernährte Nadelbaüme. Annales de Edafologia y Agrociologia, XXVI (1-4), 691-702.

LABROUE L., 1976. Etude écologique des sols alpins du Pic du Midi de Bigorre. Thèse Doctorat d'Ełat, Ecologie, Université Paul-Sabatier, Toulouse, n 717.

LEMEE G., 1967. Investigations sur la minéralisation de l'azote et son évolution annuelle dans des humus forestiers in situ. Oecol. Plant., 2, 284-324.

RUNGE M., 1974. Die Stickstoffmineralisation im Boden eines Sauerhumus-Buchenwaldes. I. Mineralstickstoffgehalt und Nettomineralisation. II. Die Nitratproduktion. Oecol. Plant., 9, 201-218 ef 219-230.

POPOVIC B., 1971. Effect of sampling date on nitrogen mineralization during incubation experiments. Plont and Soil, 34, 381-392.

VAN PRAAG H. J., 1971. Contribution à l'étude de la disponibilité de l'azote dans les sols forestiers oligotrophes de l'Ardenne. Thèse Doctorat ès Sciences agronomiques, Gembloux.

VAN PRAAG A. J., WEISSEN F., 1973. Elements of a functionnal definition of oligotroph humus based on the nitrogen nutrition of forest stands. J. Appl. Ecol., 10, 569-583. 